\title{
Géneros textuales mercantiles en la traducción jurídico-económica (alemán-español)
}

\author{
Ingrid Cobos López \\ Universidad de Córdoba \\ icobos@uco.es \\ https://dx.doi.org.10.12795/futhark.2016.il I.02
}

Fecha de recepción: II.01.2016

Fecha de aceptación: 5.03.2016

Resumen: El Derecho Mercantil es una rama del Derecho que pertenece tanto al ámbito jurídico de la traducción, como al económico. Basándonos en las teorías de los géneros textuales y en la legislación vigente, trataremos de delimitar este subgénero textual y proponer una clasificación propia de los textos que se crean dentro de este ámbito jurídico-comercial y, además, profundizar en los textos que se producen en la constitución de una sociedad mercantil en el par de lenguas alemán-español. Para ello, realizaremos nuestra propuesta y presentaremos algunos de los textos que más se traducen en este par de lenguas.

Palabras clave: género textual, textos jurídico-económicos, derecho mercantil.

\section{Mercantile genres in legal-economic translation (German-Spanish)}

\begin{abstract}
Commercial Law is the branch of law that belongs to both, the legal and the economic field of translation. Based on the theories of textual genres and on the existing legislation, we will try to define this textual subgenre and to propose our own classification of the texts that are created within this legal-commercial field. Specifically, we will look into the texts that are produced in the constitution of a commercial company in German and Spanish. To that end, we will give our proposal and we will show which are the texts that we translate the most in these two languages
\end{abstract}

Key words: textual genre, legal-economic texts, commercial law. 
Sumario: Introducción. I. El Derecho Mercantil como área de conocimiento. I.I. Fuentes del Derecho Mercantil. 2. Géneros textuales: géneros mercantiles. Conclusiones

\section{Introducción}

Existen gran cantidad de estudios sobre los géneros textuales; desde sus orígenes con Reis (197I, 1976) o Reiss \& Vermeer (1984), Göpferich (1995), hasta los últimos estudios que son referentes en la definición de dicho concepto, como son los de Gamero $(1998,200 \mathrm{I})$ o Borja (2003). Del mismo modo, observamos que hay diversas clasificaciones, en función de la perspectiva desde la que se hayan estudiado, como la de Fernández (2004) por parte de la Textología Contrastiva alemana, o la de Swales (1990) o Bhatia (1993) desde el punto de vista del Inglés para Fines Específicos.

Por otra parte, y con respecto a la traducción jurídico-económica, podemos afirmar que existen tantas acepciones, tipologías y géneros, como autores las han estudiado. Así, Alcaraz (2000), Mayoral (2000) o Borja (1999) nos ofrecen distintas definiciones y clasificaciones en función de sus perspectivas. En el presente estudio, pretendemos profundizar en una de las ramas del Derecho, como lo es el Derecho Mercantil y analizar los documentos que son necesarios para la constitución de una sociedad mercantil en España y Alemania.

Con el objetivo de proponer una clasificación de dichos textos mercantiles en base al género textual, en primer lugar, delimitaremos brevemente algunos conceptos básicos del Derecho Mercantil, para a continuación revisar el concepto de género jurídico brevemente y exponer los textos objeto de estudio.

\section{El Derecho Mercantil como área de conocimiento}

Según Sánchez Calero (2006: 42), podríamos definir el Derecho Mercantil como "la parte del Derecho Privado que comprende el conjunto de normas jurídicas relativas al empresario y a los actos que surjan del ejercicio de su actividad económica".

Tiene sus orígenes en la Edad Media. Fueron los mercaderes de la época los que lo crearon para regular o normalizar las diferencias que existían entre ellos y el ejercicio de sus actividades profesionales. En aquel momento era un derecho subjetivo y siguió siéndolo hasta el S. XIX, momento en que la Revolución francesa propició con sus ideas revolucionarias un cambio hacia la codificación mercantil. En ese momento, apareció el Código de Comercio francés de 1804 en el que se regulaba y definían las operaciones de comercio en sí mismas con independencia de los sujetos que las realizaran. 
Igualmente hemos de tener en cuenta el hecho de que, según algunos autores como Ayala Pérez ${ }^{1}$, aún hoy en día no se han conseguido definir los límites del "acto objetivo de comercio" y por ese motivo se sigue hablando tanto de derecho Mercantil como de Derecho Empresarial, términos que hemos de tomar como sinónimos. En este sentido, Uría (1997) define Derecho Mercantil o Empresarial como "el derecho ordenador de la organización y de la actividad profesional de los empresarios" (citado por Morales, 2007, p.42) .

Por todo lo anterior, resulta necesario conocer las fuentes de las que bebe dicha rama del Derecho, pues será donde el traductor deba documentarse a la hora de realizar su traducción.

\section{I.I. Fuentes del Derecho Mercantil}

Las fuentes principales del Derecho Mercantil son la Ley Mercantil y el Código de Comercio. En el propio Código de Comercio, se expone a tal fin:

Los actos de comercio..., se regulan por las disposiciones contenidas en este Código; en su defecto, por los usos de comercio observados generalmente en cada plaza; y a falta de ambas reglas, por las de Derecho común. (Código de Comercio, Art. 2) ${ }^{2}$

Existen además otras leyes de igual importancia, como son la Ley Hipotecaria Naval, la de Suspensión de Pagos, etc. que van surgiendo y se van modificando de forma constante. Debido a estas modificaciones, cuando se plantea un caso para el que no existe legislación, se aplican los usos de comercio, que viene a significar la costumbre que ha adquirido fuerza de ley. $Y$ en el caso de que no existiera ni legislación, ni uso, se aplicarían los Principios Generales del Derecho.

Igualmente cabe destacar algunas fuentes muy discutidas como son la Jurisprudencia y la Doctrina Científica, debido a que la mayoría de las empresas fueron estableciendo contratos tipo en los que fijaban sus condiciones generales de contratación en las que se han basado y se basan hoy en día tanto la misma empresa como otras independientes. Por este motivo, se plantea el hecho de tomar estos contratos y esta tendencia como Fuente de Derecho.

Como podemos observar, esta rama del Derecho, pese a estar relacionada con el ámbito económico o de comercio, se fundamenta en los códigos y la legislación, por lo que en nuestro trabajo nos centraremos en dicha parte del derecho mercantil y en los géneros textuales que se producen en dicho ámbito.

I Disponible en: https://es.scribd.com/document//3094/95/En-Forma-General-Puede-Afirmarse-QueNuestro-Codigo-de-Comercio-Delimita-La-Materia-Mercantil-en-Funcion-de-Los-Actos-CalificadosLegalmente-Como-Acto) [Fecha de consulta: noviembre 20I5].

${ }^{2}$ Disponible en: www.noticias.jurídicas.com [Fecha de consulta: diciembre 2015] 


\title{
2. Géneros textuales: géneros mercantiles
}

Como hemos indicado con anterioridad, el concepto de género se ha ido delimitando a lo largo de este último siglo y, aún a fecha de hoy, sigue siendo objeto de estudio de los académicos. El presente artículo toma como referente la clasificación realizada por Hurtado y Borja $(1998,2000)$ que clasifica dichos textos jurídicos en función de la categoría supragenérica, la función y el tono. Sin embargo, en nuestro estudio, pretendemos dar un paso más allá en dicha clasificación, y centrarnos en la situación comunicativa y/o temática de uno de los ámbitos del Derecho concretos, el Derecho Mercantil, y de forma más precisa aún, proponer, a partir de ahí, una clasificación de dicho género en el ámbito de la constitución de las sociedades mercantiles en el par de lenguas alemán-español, basándonos en las variables que determina R. Mayoral (2007) ${ }^{3}$ para este tipo de traducción.

Desde el punto de vista jurídico, podemos afirmar que las actividades mercantiles han ido creciendo a lo largo de la historia y su complejidad se ha acrecentado en los últimos años. Hoy en día, toda actividad mercantil requiere un documento escrito en el que se consignan las operaciones efectuadas de diferente índole. Los documentos mercantiles comprenden no solo a aquellos que tienen un efecto jurídico, sino que incluyen igualmente a todos aquellos relacionados con el intercambio de información, como por ejemplo la correspondencia bancaria, las anotaciones en libros de contabilidad, manuales de Derecho mercantil, etc. Nuestro propio Código de Comercio regula este hecho documental:

Las obligaciones mercantiles y su liberación se prueban:

a) con documentos públicos,

b) con documentos privados,

\begin{abstract}
${ }^{3}$ Dicho autor, (Mayoral 2007: 34), plantea la distinción entre los conceptos de traducción comercial, traducción jurídica y propone que la traducción mercantil es un sinónimo exacto de la traducción comercial: "Dentro del ámbito que podemos considerar como propiamente mercantil, se pueden producir formas de comunicación interlingüística sumamente distintas, recogiendo prácticamente cualquier modalidad que podamos imaginar: correspondencia, traducción escrita, traducción a vista, interpretación oral en todas sus modalidades (bilateral; consecutiva; susurrada; raramente, la simultánea) y por todos los medios (presencial, por teléfono, por videoconferencia, por presentaciones de PowerPoint o similares), además de comunicación y redacción monolingüe en ambas lenguas. En ocasiones, el traductor también se ve inmerso en la traducción de materiales didácticos o formativos. La traducción se hace con distintas finalidades: (I) para que surta efecto legal (jurada) ○ (2) sin efecto legal (no jurada), con carácter meramente informativo. Se hace tanto directa como inversa. Se debe estar dispuesto a trabajar en más de una lengua extranjera con un cliente (realmente ningún cliente va a entender que un traductor de inglés no separa trabajar en varias lenguas romances, oralmente o por escrito y en ambas direcciones). Las exigencias de calidad pueden ser radicalmente distintas para distintas actividades (en la actividad comercial la calidad habitual del inglés utilizado suele ser pobre pues la intervención del traductor/intérprete profesional es muy escasa)."
\end{abstract}


c) con facturas aceptadas,

d) con telegramas,

e) con cualquier otro medio de prueba admitido por la ley civil. (Código de Comercio, Art. 124)

Dentro de la documentación mercantil, tenga o no efecto legal, podemos encontrar textos de distinta índole, como por ejemplo, manuales dirigidos a estudiantes de derecho, folletos informativos del Registro Mercantil, certificados del R.M., normativa en cuanto a la constitución de sociedades, manuales para especialistas, etc.

A modo de introducción en el área de conocimiento, nos vamos a centrar en los textos relacionados con el Derecho Mercantil, y sobre todo los relacionados con el Registro Mercantil, debido a que en España existe una gran demanda en la traducción de estos géneros textuales, puesto que son los necesarios para la constitución de una sociedad mercantil. Dentro de este apartado, y basándonos en la experiencia profesional de la que disponemos, podemos distinguir distintos tipos de textos para la constitución de una sociedad:

-Textos relacionados con la actividad jurídica de la sociedad:

- Escritura de Constitución de una sociedad

- Acta de acuerdos sociales

- Orden del día de la Junta General de Accionistas

- Certificado de acuerdos sociales

- Estatutos de la sociedad

—Documentos que demanda el R. M.

- Certificado de denominación social

- Certificado de CIF

- Certificado de nombramiento del Administrador

A continuación, vamos a presentar algunos de los textos relacionados con la actividad jurídica de la sociedad, a modo de ejemplo, tanto en alemán como en español.

${ }^{4}$ íbid. 
— Escritura de constitución de una sociedad:

Texto en español:

ESCRITURA DE CONSTITUCIÓN DE SOCIEDAD DE RESPONSABILIDAD
LIMITADA - REAL DECRETO I3/20I0 NUMERO

En Almoradí, mi residencia, a *.

Ante mí, LUÍS LORENZO SERRA, Notario del Ilustre Colegio de Valencia, ----

\section{COMPARECEN*:}

He procurado su identificación mediante sus reseñados documentos.- Les juzgo capaces para formalizar la presente escritura de CONSTITUCIÓN DE SOCIEDAD DE RESPONSABILIDAD LIMITADA, a cuyo efecto,

EXPONEN*:

PRIMERO.- CONSTITUCIÓN Y ESTATUTOS DE "*, SOCIEDAD LIMITADA".

Los comparecientes tienen la voluntad de constituir y constituyen mediante esta escritura, como únicos socios fundadores, una sociedad de responsabilidad limitada al amparo del Real Decreto Ley $13 / 2010$, de 3 de Diciembre, con la denominación ya indicada, la duración indefinida, el domicilio, el objeto, el capital, los órganos y las demás determinaciones que constan en los estatutos seguidamente reseñados.

Esta sociedad se regirá especialmente por los Estatutos ajustados al modelo tipo establecido por la Orden del Ministerio de Justicia 3185/2010 - BOE de II de Diciembre de 2010 - que los comparecientes me entregan para su protocolización y que se hallan extendidos en $*$ folios de papel exclusivo para documentos notariales, serie *, números *.

En este acto, yo, el Notario, leo dichos Estatutos a los comparecientes, quienes los aprueban y los firmarán al final de su último folio.- En su consecuencia, los repetidos Estatutos quedan unidos a esta escritura matriz, como parte integrante de la misma.

Fuente: https://www.notariosyregistradores.com/PERSONAL/NOTARIAS/documentos/20II modelosnuevassociedades.htm\#modelol. 


\section{SEGUNDO.- CAPITAL, SUSCRIPCIÓN Y DESEMBOLSO DE LAS PARTICIPACIONES SOCIALES}

El capital social queda fijado en * EUROS y dividido en * PARTICIPACIONES SOCIALES iguales, numeradas correlativamente a partir de la unidad, para el solo efecto de su identificación, y de un valor nominal de * EUROS cada una.---

Los socios fundadores suscriben la totalidad de las participaciones sociales y desembolsan íntegramente su valor nominal en la forma seguidamente determinada:-

A) D. * suscribe * participaciones sociales, las números del * al *, ambos inclusive, cuyo total valor asciende a * euros, y aporta esta cantidad para el dicho desembolso íntegro, acreditando lo anterior con certificación de depósito de la referida cantidad en la Entidad Bancaria *, de fecha *, que incorporo a esta matriz.-----

B) D. * suscribe * participaciones sociales, las números del $*$ al $*$, ambos inclusive, cuyo total valor asciende a * euros, y aporta esta cantidad para el dicho desembolso íntegro, acreditando lo anterior con certificación de depósito de la referida cantidad en la Entidad Bancaria *, de fecha *, que incorporo a esta matriz.----.

C) Y D. * suscribe * participaciones sociales, las números del * al *, ambos inclusive, cuyo total valor asciende a $*$ euros, y aporta esta cantidad para el dicho desembolso íntegro, acreditando lo anterior con certificación de depósito de la referida cantidad en la Entidad Bancaria *, de fecha *, que incorporo a esta matriz.-----

(...)

Del íntegro contenido de esta diligencia extendida en *, yo, el Notario, DOY FE.-

Texto en alemán ${ }^{6}$ :

Öffentliche Beurkundung

Gründung

der

* (Name der Gesellschaft)

mit Sitz in * (Sitz der Gesellschaft)

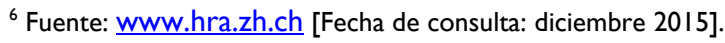


Im Amtslokal des Notariates * (Name des Notariates) sind heute er-schienen:

I.* (Vorname, Name, Geburtsdatum, schweizerischer Bürgerort oder ausländische Staatsangehörigkeit und Wohnadresse);

2.* (dito);

[Bemerkung: Hinweis auf allfällige Vertretungsverhältnisse sowie bei juristischen Personen oder anderen Handelsgesellschaften auf deren Firma, Rechtsform und Sitz (gegebenenfalls Staat). Die entsprechen-den, vorliegenden Belege, wie beglaubigte Vollmachten, Handels-registerauszüge, sind in der Urkunde einzeln zu nennen.

und erklären:

I.

Unter der Firma

* (Name der Gesellschaft)

gründen wir gemäss den Bestimmungen des Schweizerischen Obligationenrechtes (OR) eine Gesellschaft mit beschränkter Haftung mit Sitz in * (Sitz der Gesellschaft).

II.

Den uns vorliegenden Statutenentwurf legen wir als gültige Statuten der in Gründung begriffenen Gesellschaft fest. Sie sind Bestandteil dieser Urkunde.

(Erläuterung siehe hinten)

III.

Das Stammkapital der Gesellschaft beträgt CHF * (Stammkapital) und ist eingeteilt in * (Anzahl, gegebenenfalls Kategorie der Stammanteile, z.B. Stimmrechts- oder Vorzugs-Stammananteile) Stammanteile zu je CHF * (Nennwert), welche zum Ausgabebetrag von CHF * (mindestens zum Nennwert) je Stammanteil wie folgt gezeichnet werden:

a)* Stammanteile von * (Vorname, Name des einen Gründers)

b) $*$ Stammanteile von * (Vorname, Name des andern Gründers)

* Stammanteile total

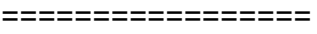

$(\ldots)$ 
Como podemos observar, se trata de una escritura notarial que tiene una estructura muy similar en alemán y español y que están fuertemente convencionalizadas, rasgos propios de los géneros que señala Borja (2007).

-Acta de acuerdos sociales:

Texto en español':

Acta de la Junta General Ordinaria o Extraordinaria de una sociedad mercantil

Este documento está destinado a los socios de una sociedad limitada (SL o SRL) y a los accionistas de una Sociedad Anónima (SA) que deseen redactar el acta de la Junta general ordinaria u extraordinaria, universal $\circ$ no, de su sociedad.

El documento se adapta a las diferentes formas de Junta General: universal y no universal, ordinaria y extraordinaria. Sin embargo, solo se aplica a las sociedades limitadas $\circ$ anónimas que tienen varios socios. Para las sociedades unipersonales, es necesario levantar un Acta de decisiones del socio o accionista único.

Acuerdos incluidos:

Este documento tiene en consideración los siguientes acuerdos:

la aprobación de las cuentas anuales del ejercicio anterior;

la aplicación del resultado del ejercicio anterior;

la aprobación de la gestión social del ejercicio anterior;

el nombramiento, cese o reelección de los administradores;

la aprobación del importe máximo de la remuneración anual de los administradores;

el nombramiento de los auditores de cuentas;

la aprobación de la transmisión voluntaria de las participaciones sociales a una persona ajena a la sociedad en el caso de las sociedades limitadas; $\boldsymbol{y}$,

la modificación de los estatutos de la sociedad.

Además, el documento permite personalizar el acta, pudiendo incluirse otros acuerdos adoptados por la Junta General

7 Fuente: https://www.wonder.legal/es/modele/acta-junta-general-ordinaria-extraordinariasociedad-limitada. [Fecha de consulta: enero 2016]. 
Texto en alemán 8 :

$$
\begin{gathered}
\text { Gesellschafterbeschluss } \\
\text { Vorabausschüttung - Niederschrift }
\end{gathered}
$$

Zur Gesellschafterversammlung der: am sind erschienen:

Gesellellschafter, mit einer Beteiligung von

I. Feststellung der ordnungsgemäßen Ladung

Die Gesellschafterversammlung stellt fest:

I. Die heutige Gesellschafterversammlung wurde ordnungsgemäß, unter Verzicht auf alle Form

und Fristregelungen, einberufen.

2. Das Stammkapital der Gesellschaft ist in voller Höhe vertreten. Die Versammlung ist somit

beschlussfähig.

II. Beschlussfassung

Danach beschließt die Gesellschafterversammlung im Wege mündlicher Abstimmung wie folgt:

Die Geschäftsführung hat den Gesellschaftern den aufgestellten Jahresabschluss für das Geschäftsjahr __ vorgelegt. Der aufgestellte Jahresabschluss wird hiermit festgestellt. Der Geschäftsführung wird Entlastung erteilt.

Der Jahresüberschuss/Jahresfehlbetrag wird auf neue Rechnung vorgetragen.

Der Beschluss wird einstimmig gefasst.

En el caso de las actas de acuerdos sociales, debemos poner de manifiesto que existe una gran variedad de tipos de actas, puesto que en ellas se recogen los acuerdos a los que llegan los socios, en función de la temática del acto constitutivo en sí, así pues podemos encontrar cambios de administrador, aumentos de capital, poderes, aprobación de balances, revisiones de cuentas, etc.

${ }^{8}$ Fuente: www.steuerberater-aichach.de [Fecha de consulta: diciembre 2015]. 
-Orden del día de la Junta General de Accionistas:

Texto en español':

Convocatoria Junta General de Accionistas

El Consejo de Administración de FENIE ENERGÍA, S.A., con ..., ha acordado convocar Junta General Ordinaria de Accionistas, que se celebrará en el La Ciudad del Fútbol de las Rozas, (Calle Ramón y Cajal, s/n, 28.230 Las Rozas de Madrid) el día ... horas en primera convocatoria, ..., a la misma hora, en segunda convocatoria, para el caso de que, por no haberse alcanzado el quorum necesario, dicha Junta no pueda celebrarse en primera convocatoria, con el fin de que los Señores Accionistas puedan deliberar y resolver sobre los puntos del siguiente:

Orden del día

Primero.- Informe del Presidente y de actividad de la Compañía;

Segundo. - Examen y aprobación, en su caso, de las cuentas anuales de la Sociedad correspondiente al ejercicio cerrado a 3I de diciembre de 2015 compuestas por balance, cuenta de pérdidas y ganancias, estado de patrimonio neto del ejercicio y memoria;

Tercero. - Aprobación, en su caso, de la propuesta de aplicación del resultado correspondiente al ejercicio 2015; Cuarto. - Aprobación de la gestión y actuación del Consejo de Administración durante los ejercicios 2013, 2014 y 2015.

Quinto. - Aprobación, si procede, de la modificación del artículo 2 de los Estatutos de la Sociedad sobre objeto social;

Sexto. - Aprobación, si procede, de la modificación del artículo 6 de los Estatutos de la Sociedad sobre domicilio social;

Séptimo. - Aprobación, si procede, de la modificación del artículo 7 de los Estatutos de la Sociedad sobre pérdida de los requisitos para ser titular de acciones $\mathrm{A}$ y $\mathrm{B}$, según el caso;

$(\ldots)$ 
Texto en alemán ${ }^{10}$ :

EINLADUNG ZUR

\section{GENERALVERSAMMLUNG}

Mittwoch, 18. Juni 2014

um 19.30 Uhr bzw.

nach Ablauf einer Wartehalbestunde um 20.00 Uhr im Gasthof Neuwirt, 6382

Kirchdorf, Wenger Straße 10

Tagesordnung

I.Eröffnung und Begrüßung durch den Vorsitzenden,

2.Feststellung der ordnungsgemäßen Einberufung und der Beschlussfähigkeit,

Ernennung des Protokollführers, Wahl des Protokollmitfertigers und zweier Stimmenzähler

3.Vorlage des Geschäftsberichtes 2013 durch den Vorstand

4. Vorlage des Jahresabschlusses 2013 und des Lageberichtes

5.Bericht, über die gesetzliche Revision und Jahresabschlussprüfung 2013

6.Bericht des Aufsichtsrates

7.Beschlussfassung, der

- Behandlung des Revisionsberichtes

- Genehmigung des Jahresabschlusses 2013

- Verwendung des Bilanzgewinnes

- Entlastung des Vorstandes und des Aufsichtsrates

8.Wahlen in den Aufsichtsrat

- turnusmässig scheidet aus:

Vorsitzender-Stellvertreter

- $\quad$ turnusmässig scheidet aus:

Aufsichtsratsmitglied

9.Anfragen und Mitteilungen

(..)

${ }^{10}$ Fuente: https://www.raiffeisen.at [Fecha de consulta: diciembre 2015] 
En el caso del orden del día, observamos que se trata de un documento más administrativo y que están fuertemente convencionalizado en ambos idiomas.

\section{-Estatutos de la sociedad:}

Texto en español":

TíTULO I.- DENOMINACIÓN, DURACIÓN, DOMICILIO Y OBJETO

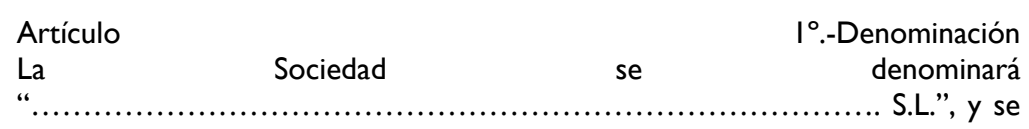
regirá por su contrato constitutivo, por los presentes estatutos y en lo en ellos no dispuesto, por la Ley de Sociedades de Capital.

Artículo

$2^{\circ}$.-Duración

La Sociedad tendrá una duración de carácter indefinido, dándose comienzo al inicio de sus operaciones el día siguiente al del otorgamiento de su escritura constitutiva, sin perjuicio de las consecuencias legales previstas para los actos y contratos celebrados en nombre de la Sociedad en momentos anteriores al de su inscripción en el Registro Mercantil.

Los ejercicios sociales se computarán por años naturales.

\begin{tabular}{|c|c|}
\hline $\begin{array}{l}\text { Artículo } \\
\text { El domicilio de la Sociedad se halla situado en } \\
\text { calle } \ldots \ldots \ldots \ldots \ldots \ldots \ldots \ldots \ldots \text { número } \ldots \ldots \ldots \ldots . . . \ldots\end{array}$ & $3^{\circ}$.-Domicilio \\
\hline $\begin{array}{ll}\text { Artículo } & 4^{\circ} \text {.-Objeto } \\
\text { El objeto social de la Sociedad será } & \ldots \ldots \ldots \ldots . . .\end{array}$ & SOC \\
\hline
\end{tabular}

TÍTULO II.- CAPITAL SOCIAL Y PARTICIPACIONES

$(\ldots)$

" Fuente: www.crear-empresas.com [fEcha de consulta: diciembre 2015]. 
Texto en alemán ${ }^{12}:$

SATZUNG DER DEUTSCHEN GESELLSCHAFT FUR KRISTALLOGRAPHIE (DGK)

STAND: 18.03 .2014

Präambel

§। Name, Sitz, Geschätsjahr

$\S 2$ Zweck der Gesellschaft

$\S 3$ Gemeinnützigkeit

$\S 4$ Mitgliedschaft

$\S 5$ Einhaltung guter wissenschaftlicher Praxis

\$6 Rechte und Pflichten der Mitglieder

$\S 7$ Mitgliedsbeiträge und Finanzierung

$\S 8$ Organe der Gesellschaft

$\S 9$ Die Mitgliederversammlung

$\S 10$ Der Vorstand

§II Der engere Vorstand

$\S 12$ Wahlen zum Vorstand

§13 Nationalkomitee für Kristallographie

§14 Der Beirat

$\S 15$ Arbeitskreise und Kommissionen

§16 Assoziierte Gesellschaften

\$17 Carl-Hermann-Medaille

$\S 18$ Will-Kleber-Gedenkmünze

$\$ 19$ Max-von-Laue-Preis

\$20 Änderungen des Statutes und Auflösung der DGK

$\S 21$ Haftung

Präambel

${ }^{12}$ Fuente: www.dgk-home.de [Fecha de consulta: diciembre 2015]. 
Die Gründung der Deutschen Gesellschaft für Kristallographie e.V. (DGK) stellt die organisatorische Weiterentwicklung der wissenschaftlichen Vereinigungen „Arbeits- gemeinschaft Kristallographie“ (AGKr) und „Vereinigung für Kristallographie“ (VFK) dar.

§। Name, Sitz, Geschaftsjahr

Die Gesellschaft trägt den Namen ,Deutsche Gesellschaft für Kristallographie e. V.“ (DGK) und ist im Vereinsregister eingetragen.

Die DGK ist eine juristische Person und hat ihren Sitz in Berlin.

Das Geschaftsjahr ist das Kalenderjahr.

\section{(...)}

Este es quizás el documento más completo de los que podemos encontrar en los acuerdos sociales y el primero que se ha de redactar a la hora de constituir una sociedad. De ahí que encontremos toda la terminología específica del ámbito del Derecho Mercantil y la legislación vigente relativa al tipo societario concreto. Es, por tanto, un texto altamente convencionalizado y que posee una macroestructura y microestructura muy estereotipada.

\section{Conclusiones}

Una vez revisada la bibliografía sobre los géneros textuales y más en concreto sobre los géneros jurídicos y tras haber considerado la legislación existente entorno al Derecho Mercantil por el que se regulan los hechos documentales de este ámbito, observamos que, dentro de los géneros mercantiles, encontraremos textos de temática jurídica y de temática económica. De ahí, la dificultad existente a la hora de definirlos y catalogarlos en un tipo de traducción especializada. Si bien es cierto que autores como Mayoral (2007) los incluyen dentro de los documentos comerciales, hemos podido comprobar que igualmente encontramos textos que se enmarcan exclusivamente en el ámbito jurídico, como lo son aquellos relacionados con la constitución de la sociedad mercantil.

En el par de lenguas alemán-español, observamos gran similitud entre los textos seleccionados que es debida a las fuentes de las que se nutre nuestro Derecho Mercantil.

Consideramos pues, que este género textual, el género mercantil, fluye entre la traducción jurídica y económica y que deberíamos realizar, en futuras investigaciones, una clasificación más completa de todo el subgénero textual y analizar los textos que se generan en cada uno de ellos de forma más completa. 


\section{Referencias bibliográficas}

ALCARAZ, E. (2000). El inglés Profesional y Académico. Madrid: Alianza.

BHATIA, V. K. (1993). Analysing genre. Language use in professional settings. Londres: Longman.

BercovitZ, A. (2008). Apuntes de Derecho Mercantil. Pamplona: Aranzadi.

BORJA, A. (1999). La traducción jurídica en España al filo del milenio: profesión e investigación. En Perspectives, Studies in Translatology, 154-166.

FERNÁNDEZ, F. (2004). El folleto de cursos de idiomas para extranjeros: análisis contrastivo (alemán-español) por tipos de emisor y subtextos. Tesis doctoral. Barcelona: Universitat Pompeu Fabra.

GAMERO, S. (200I). La traducción de textos técnicos. Madrid: Ariel Lenguas modernas.

GÖPFERICH, S. (1995). Textsorten in Naturwissenschaften und Technik. Pragmatische Typologie - Kontrastierung - Translation. Túbingen: Narr.

HurTADO, A., (200I). Traducción y traductología. Introducción a la traductología. Madrid: Cátedra.

MAYORAL, R. (2000). ¿Cómo se hace la traducción jurídica? [Disponible en http://www.gitrad.uji.es/bibliografia/bidvirtual Fecha de consulta: I5 mayo 20I4].

(2007). La traducción comercial. En P. A. Fuertes Olivera (eds.), Problemas lingüisticos en la traducción especializada. Valladolid: Universidad de Valladolid, 33-48.

Morales, A. (2007). Curso de Derecho Mercantil: Introducción La empresa EI empresario. Montalbán, Caracas: Editorial Texto, C.A.

REISS, K. (197I). Möglichkeiten und Grenzen der Übersetzungskritik. Múnich: Hueber. . (1976). Texttyp und Übersetzungsmethode. Der operative Text. Scriptor Verlag.

REISS, K. y VERMEER H. J. (1984). Grundlegung einer Allgemeinen Translationstheorie. Tuibingen: Niemeyer. (Traducción al español de S. García Reina y C. Martín de León. 1996. Fundamentos para una teoría funcional de la traducción. Madrid: Akal).

Sánchez, F. y Sánchez-Calero, J. (2006). Instituciones de Derecho Mercantil I. Navarra: Aranzadi.

SWALes, J. M. (1990). Genre Analysis. English in Academic and Research Settings. Cambridge: Cambridge University Press

SAPIR, E. (I92I). Language: an introduction to study of speech. New York: Harcourt, Brace and company.

Recursos Web

http://noticias.juridicas.com/base_datos/Privado/ccom.lltl.html. (Código de Comercio Español de 1885). 
https://es.scribd.com/document//3094 I95/En-Forma-General-Puede-Afirmarse-

Que-Nuestro-Codigo-de-Comercio-Delimita-La-Materia-Mercantil-en-

Funcion-de-Los-Actos-Calificados-Legalmente-Como-Acto

www.notariosyregistradores.com

www.hra.zh.ch

www.wonder.legal.es

www.steuerberater-aichach.de

www.fenieenergia.es

www.raiffeisen.at

www.crearempresas.com

www.dgk-home.de 
